\title{
Boundary Value Problems for Nonlinear Elliptic Equations of Second Order in High Dimensional Domains
}

\author{
Guochun Wen ${ }^{1}$, Dechang Chen ${ }^{2}$ \\ ${ }^{1}$ School of Mathematical Sciences, Peking University, Beijing, China \\ ${ }^{2}$ Uniformed Services University of the Health Sciences, Bethesda, USA \\ Email: wengc@math.pku.edu.cn, dechang.chen@usuhs.edu
}

Received September 17, 2013; revised October 17, 2013; accepted October 24, 2013

Copyright (C) 2013 Guochun Wen, Dechang Chen. This is an open access article distributed under the Creative Commons Attribution License, which permits unrestricted use, distribution, and reproduction in any medium, provided the original work is properly cited.

\section{ABSTRACT}

This paper mainly concerns oblique derivative problems for nonlinear nondivergent elliptic equations of second order with measurable coefficients in a multiply connected domain. Under certain condition, we derive a priori estimates of solutions. By using these estimates and the fixed-point theorem, we prove the existence of solutions.

Keywords: Oblique Derivative Problems; Nonlinear Elliptic Equations; High Dimensional Domains

\section{Formulation of Oblique Derivative Problems for Nonlinear Elliptic Equations of Second Order}

Let $Q$ be a bounded domain in $\mathbb{R}^{N}$ with the boundary $\partial Q \in C_{\alpha}^{2}(0<\alpha<1)$. We consider the nonlinear elliptic equation of second order

$$
F\left(x, u, D_{x} u, D_{x}^{2} u\right)=0 \text { in } Q,
$$

where $u=u(x)=u\left(x_{1}, \cdots, x_{N}\right)$,

$D_{x} u=\left(u_{x_{i}}\right), D_{x}^{2} u=\left(u_{x_{i} x_{j}}\right)$. Under certain conditions, the above equation can be rewritten in the form

$$
F\left(x, u, D_{x} u, D_{x}^{2} u\right)=\sum_{i, j=1}^{N} a_{i j} u_{x_{i} x_{j}}+\sum_{i=1}^{N} b_{i} u_{x_{i}}+c u-A=0 \text { in } Q,
$$

where

$$
\begin{aligned}
& a_{i j}=\int_{0}^{1} F_{\tau r_{i j}}(x, u, p, \tau r) \mathrm{d} \tau, b_{i}=\int_{0}^{1} F_{\tau p_{i}}(x, u, \tau p, 0) \mathrm{d} \tau, \\
& c=\int_{0}^{1} F_{\tau u}(x, \tau u, 0,0) \mathrm{d} \tau, A=-F(x, 0,0,0),
\end{aligned}
$$

with

$$
p=D_{x} u, r=D_{x}^{2} u, r_{i j}=u_{x_{i} x_{j}} .
$$

In this paper, the notations are the same as in References [1-8]. The main equation to be studied in this paper is

$$
\sum_{i, j=1}^{N} a_{i j} u_{x_{i} x_{j}}+\sum_{i=1}^{N} b_{i} u_{x_{i}}+c u+G\left(x, u, D_{x} u\right)=A \text { in } Q .
$$

Suppose that (1.2) satisfies the following conditions.

Condition C. For arbitrary functions $u^{1}=u^{1}(x)$, $u^{2}=u^{2}(x) \in C_{\beta}^{1}(\bar{Q}) \cap W_{2}^{2}(Q) \quad(0<\beta<1)$, let $u=u^{1}-u^{2}$. Then $F\left(x, u, D_{x} u, D_{x}^{2} u\right)=\sum_{i, j=1}^{N} a_{i j} u_{x_{i} x_{j}}+\sum_{i=1}^{N} b_{i} u_{x_{i}}+c u-A$ satisfies the following:

$$
\begin{aligned}
& F\left(x, u^{1}, D_{x} u^{1}, D_{x}^{2} u^{1}\right)-F\left(x, u^{2}, D_{x} u^{2}, D_{x}^{2} u^{2}\right) \\
& =\sum_{i, j=1}^{N} \tilde{a}_{i j} u_{x_{i} x_{j}}+\sum_{i=1}^{N} \tilde{b}_{i} u_{x_{i}}+\tilde{c} u,
\end{aligned}
$$

where

$$
\begin{aligned}
& \tilde{a}_{i j}=\int_{0}^{1} F_{u_{x_{i} x_{j}}}(x, \tilde{u}, \tilde{p}, \tilde{r}) \mathrm{d} \tau, \tilde{b}_{i}=\int_{0}^{1} F_{u_{x_{i}}}(x, \tilde{u}, \tilde{p}, \tilde{r}) \mathrm{d} \tau, \\
& \tilde{c}=\int_{0}^{1} F_{u}(x, \tilde{u}, \tilde{p}, \tilde{r}) \mathrm{d} \tau
\end{aligned}
$$

for

$$
\tilde{r}=D_{x}^{2}\left(u^{2}+\tau u\right), \tilde{p}=D_{x}\left(u^{2}+\tau u\right), \tilde{u}=u^{2}+\tau u .
$$

Here $\tilde{a}_{i j}, \tilde{b}_{i}, \tilde{c}$ satisfy the conditions

$q_{0} \sum_{j=1}^{n}\left|\xi_{j}\right|^{2} \leq \sum_{i, j=1}^{N} \tilde{a}_{i j} \xi_{i} \xi_{j} \leq q_{0}^{-1} \sum_{j=1}^{n}\left|\xi_{j}\right|^{2}, 0<q_{0}<1$,

$\sup _{Q}\left[\sum_{i, j=1}^{N}\left(\tilde{a}_{i j}\right)^{2}\right] / \inf _{Q}\left[\sum_{i=1}^{N} \tilde{a}_{i i}\right]^{2} \leq q_{1}<\frac{2 N-1}{2 N^{2}-2 N-1}$,

$$
\begin{aligned}
& \left|\tilde{a}_{i j}\right| \leq k_{0},\left|\tilde{b}_{i}\right| \leq k_{0}, i, j=1, \cdots, N,|\tilde{c}| \leq k_{0}, \\
& \sup _{Q} \tilde{c}<0, L_{p}[A, \bar{Q}) \leq k_{1},
\end{aligned}
$$


in which $q_{0}, q_{1}, k_{0}, k_{1}, p(>N+2)$ are positive constants. Moreover, for almost every point $x \in Q$ and $D_{x}^{2} u$, $\tilde{a}_{i j}\left(x, u, D_{x} u, D_{x}^{2} u\right), \quad \tilde{b}_{i}\left(x, u, D_{x} u\right), \quad \tilde{c}(x, u)$ are continuous in $u \in \mathbb{R}, \quad D_{x} u \in \mathbb{R}^{N}$.

Moreover if the Equation (1.2) satisfies Condition C and the function

$$
G\left(x, u, D_{x} u\right)=\sum_{i=1}^{N} B_{i}\left|u_{x_{i}}\right|^{\sigma_{i}}+B_{0}|u|^{\sigma_{0}}
$$

satisfies

$$
\left|B_{i}\right| \leq k_{0}, i=0,1, \cdots, N,
$$

where $k_{0}, \sigma_{i}(i=0,1, \cdots, N)$ are positive constants, then we say that the Equation (1.2) satisfies Condition $\mathrm{C}^{\prime}$.

The motivation for the second formula in (1.3) may be given as follows. It is enough to consider the linear elliptic Equation of (1.1), namely

$$
\sum_{i, j=1}^{N} a_{i j}(x) u_{x_{i} x_{j}}+\sum_{i=1}^{N} b_{i}(x) u_{x_{i}}+c(x) u=A(x) \text { in } Q .
$$

Let (1.6) be divided by $\Lambda=\tau \inf _{Q} \sum_{i=1}^{N} a_{i i}$, where $\tau$ is an undetermined positive constant, and denote $\hat{a}_{i j}=a_{i j} / \Lambda, \hat{b}_{i}=b_{i} / \Lambda(i, j=1, \cdots, N), \hat{c}=c / \Lambda, \hat{A}=A / \Lambda$. Then the Equation (1.6) is reduced to the form

$$
\begin{gathered}
\sum_{i, j=1}^{N} \hat{a}_{i j}(x) u_{x_{i} x_{j}}+\sum_{i=1}^{N} \hat{b}_{i}(x) u_{x_{i}}+\hat{c}(x) u=\hat{A}(x), \text { i.e. } \\
\Delta u=\sum_{i=1}^{N} \partial^{2} u / \partial x_{i}^{2}=-\sum_{i, j=1}^{N}\left[\hat{a}_{i j}(x)-\delta_{i j}\right] u_{x_{i} x_{j}} \\
-\sum_{i=1}^{N} \hat{b}_{i}(x) u_{x_{i}}-\hat{c}(x) u+\hat{A}(x) \text { in } Q .
\end{gathered}
$$

We require that the above coefficients satisfy

$$
\begin{aligned}
& \sup _{Q}\left[2 \sum_{i, j=1, i<j}^{N} \hat{a}_{i j}^{2}+\sum_{i=1}^{N}\left(\hat{a}_{i i}-1\right)^{2}\right] \\
& =\sup _{Q}\left[\sum_{i, j=1}^{N} \hat{a}_{i j}^{2}+N-2 \sum_{i=1}^{N} \hat{a}_{i i}\right]<\frac{N+1}{2 N-1}, \text { i.e. } \\
& \sup _{Q}\left[\sum_{i, j=1}^{N} \hat{a}_{i j}^{2}-2 \sum_{i=1}^{N} \hat{a}_{i i}\right]<\frac{N+1}{2 N-1}-N,
\end{aligned}
$$

which can be derived from the condition in (1.3) with the constant $\tau=(2 N-1) /\left(2 N^{2}-2 N-1\right)$. In fact, we consider

$\sup _{Q} \sum_{i, j=1}^{N} \hat{a}_{i j}^{2}-2 \inf _{Q} \sum_{i=1}^{N} \hat{a}_{i i}<\frac{N+1}{2 N-1}-N$, i.e.

$\frac{\sup _{Q} \sum_{i, j=1}^{N} a_{i j}^{2}}{\tau^{2} \inf _{Q}\left[\sum_{i=1}^{N} a_{i i}\right]^{2}}<\frac{2}{\tau}+\frac{N+1}{2 N-1}-N$, or $\frac{\sup _{Q} \sum_{i, j=1}^{N} a_{i j}^{2}}{\inf _{Q}\left[\sum_{i, j=1}^{N} a_{i i}\right]^{2}}<f(\tau)$,

for $f(\tau)=2 \tau+\left(1+2 N-2 N^{2}\right) \tau^{2} /(2 N-1)$. It is seen that the maximum of $f(\tau)$ on $[0, \infty)$ occurs at the point $\tau=(2 N-1) /\left(2 N^{2}-2 N-1\right)$, and the maximum of $f(\tau)$ equals

$$
f\left((2 N-1) /\left(2 N^{2}-2 N-1\right)\right)=(2 N-1) /\left(2 N^{2}-2 N-1\right) \text {. }
$$

The above inequality with $\tau=(2 N-1) /\left(2 N^{2}-2 N-1\right)$ is just the second inequality in (1.3), from which (1.7) with $\tau=(2 N-1) /\left(2 N^{2}-2 N-1\right)$ holds.

Problem O. The so-called Problem $\mathrm{O}$ of an oblique derivative boundary value problem is to find a continuously differentiable solution

$u=u(x) \in B^{*}=C_{\beta}^{1}(\bar{Q}) \cap W_{2}^{2}(Q)$ of the equation that satisfies the boundary conditions

$$
\begin{aligned}
& l u=\frac{\partial u}{\partial v}+\sigma u=g(x), x \in \partial Q, \text { i.e. } \\
& l u=\sum_{j=1}^{N} d_{j} \frac{\partial u}{\partial x_{j}}+\sigma u=g(x), x \in \partial Q .
\end{aligned}
$$

Here $d(x)=\left(d_{1}(x), d_{2}(x), \cdots, d_{N}(x)\right)$ represents the direction of $v$, and $\sigma(x)$ and $g(x)$ satisfy the conditions

$$
\begin{aligned}
& C_{\alpha}^{1}[\sigma(x), \partial Q] \leq k_{0}, C_{\alpha}\left[d_{j}(x), \partial Q\right] \leq k_{0}, \\
& C_{\alpha}^{1}[g(x), \partial Q] \leq k_{2}, \cos (v, \mu) \geq q_{0}>0, \quad \sigma \geq 0, x \in \partial Q,
\end{aligned}
$$

where $\mu$ is the unit outward normal on $\partial Q, \alpha(0<\alpha<1), k_{0}, k_{2}, \quad q_{0}\left(0<q_{0}<1\right)$ are non-negative constants. Noting that Problem $\mathrm{O}$ with the condition $v=\mu, \sigma=0$ on $\partial Q$ is the initial-Neumenn problem.

Theorem 1.1. If (1.2) satisfies Condition $\mathrm{C}$ and $A=0, G=0, g=0$, then Problem $O$ for (1.2) has the trivial solution.

Proof. Let $u(x)$ be one solution of Problem O. It is easy to see that $u(x)$ satisfies the following boundary value problem

$$
\begin{gathered}
\sum_{i, j=1}^{N} a_{i j} u_{x_{i} x_{j}}+\sum_{i=1}^{N} b_{i} u_{x_{i}}+c u=0, x \in Q, \\
\frac{\partial u}{\partial v}+\sigma(x) u=0, x \in \partial Q
\end{gathered}
$$

where $a_{i j}, b_{i}, c$ are as stated in (1.2). Multiplying both sides of (1.1) by $u$ we obtain the following equation on $u^{2}$ :

$$
\frac{1}{2} \sum_{i, j=1}^{N} a_{i j}\left(u^{2}\right)_{x_{i} x_{j}}-\sum_{i, j=1}^{N} a_{i j} u_{x_{i}} u_{x_{j}}+\sum_{i=1}^{N} b_{i} u u_{x_{i}}+c u^{2}=0 \text { in } Q .
$$

Noting that Condition $\mathrm{C}$, if the maximum of $u^{2}$ attains at an inner point $P_{0}=x_{0} \in Q$, and 
$M_{0}=\left|u\left(P_{0}\right)\right|^{2}>0$, there exists a small positive number $\varepsilon_{0}$ and we can choose a sufficiently small neighborhood $G_{0} \quad$ of $P_{0}$ such that $2\left|u u_{x_{i}}\right| \leq \varepsilon_{0}^{2} / N M_{0}$ in $G_{0}$ and $\left(-\sup _{Q} c\right)-\left[N / 4 q_{0}+k_{0} / M_{0}+1\right] \varepsilon_{0}^{2}>0$, thus we have

$$
\begin{aligned}
& \sum_{i, j=1}^{N} a_{i j} u_{x_{i}} u_{x_{j}} \geq q_{0} \sum_{i=1}^{N}\left|u_{x_{i}}\right|^{2}, \\
& -\sum_{i=1}^{N} b_{i} u u_{x_{i}} \geq-q_{0} \sum_{i=1}^{N}\left|u_{x_{i}}\right|^{2}-\left[\frac{N}{4 q_{0}}+\frac{k_{0}}{M_{0}}\right] \varepsilon_{0}^{2} u^{2}, \\
& -c u^{2} \geq\left[\underset{Q}{\left.\sup c-\varepsilon_{0}^{2}\right] u^{2},}\right.
\end{aligned}
$$

and then

$$
\sum_{i, j=1}^{N} a_{i j} u_{x_{i}} u_{x_{j}} \geq\left\{-\sup c-\left[\frac{N}{4 q_{0}}+\frac{k_{0}}{M_{0}}+1\right] \varepsilon_{0}^{2}\right\} u^{2}>0 \text { in } G_{0} .
$$

On the basis of the maximum principle of the solution $u^{2}$ for Equation (1.12), we see that $u^{2}$ cannot take a positive maximum in $Q$. Hence $u^{2}(x)=0$, namely $u(x)=0$ in $Q$.

\section{Estimates of Solutions of Oblique Derivative Problems for Nonlinear Elliptic Equations of Second Order}

We begin with the estimates of the solutions of (1.2) when $G=0$.

Theorem 2.1. Suppose that (1.2) with $G\left(x, u, D_{x} u\right)=0$ satisfies Condition C. Then any solution $u(x)$ of Problem $\mathrm{O}$ satisfies the estimates

$$
\begin{gathered}
C_{\beta}^{1}(u, \bar{Q}) \leq M_{1}=M_{1}(q, p, \beta, k, Q), \\
\|u\|_{W_{2}^{2}(Q)} \leq M_{2}=M_{2}(q, p, \beta, k, Q),
\end{gathered}
$$

where

$\beta(0<\beta \leq \alpha), q=\left(q_{0}, q_{1}\right), k=k\left(k_{0}, k_{1}, k_{2}\right), M_{1}, M_{2} \quad$ are non-negative constants.

Proof. After substituting the solution $u(x)$ into (1.2), we see that we only need to discuss the linear elliptic equation in the form

$$
\sum_{i, j=1}^{N} a_{i j} u_{x_{i} x_{j}}+\sum_{i=1}^{N} b_{i} u_{x_{i}}+c u=A \text { in } Q .
$$

Below we will verify the boundedness of the estimate of the solution $u(x)$ :

$$
C^{1}[u, \bar{Q}] \leq M_{3},
$$

where $M_{3}=M_{3}(q, p, \beta, k, Q)$. Suppose that (2.4) is not true, then there exist sequences of functions $\left\{a_{i j}^{(l)}\right\}$, $\left\{b_{i}^{(l)}\right\}, \quad\left\{c^{(l)}\right\}, \quad\left\{A^{(l)}\right\}$ and $\left\{\sigma^{(l)}\right\}, \quad\left\{g^{(l)}\right\}$, meeting
(1.4) and (1.9), such that $\left\{a_{i j}^{(l)}\right\},\left\{b_{i}^{(l)}\right\},\left\{c^{(l)}\right\},\left\{A^{(l)}\right\}$ weakly converge to $a_{i j}^{(0)}, b_{i}^{(0)}, c^{(0)}, A^{(0)}$, and $\left\{\sigma^{(l)}\right\}$, $\left\{g^{(l)}\right\}$ uniformly converge to $\sigma^{0}, g^{(0)}$ in $\Omega$ and $\partial Q$ respectively, and the boundary value problem

$$
\begin{gathered}
\sum_{i, j=1}^{N} a_{i j}^{(l)} u_{x_{i} x_{j}}^{(l)}+\sum_{i=1}^{N} b_{i}^{(l)} u_{x_{i}}^{(l)}+c^{(l)} u^{(l)}=A^{(l)} \text { in } Q, \\
\frac{\partial u^{(l)}}{\partial v}+\sigma^{(l)} u^{(l)}=g^{(l)}(x) \text { on } \partial Q,
\end{gathered}
$$

have solutions $u^{(l)}(x)(l=1,2, \cdots)$ with unbounded $h^{(l)}=\left\|u^{(l)}\right\|_{C^{1}(\bar{Q})}$. There is no harm assuming that $h^{(l)} \geq 1$, and $\lim _{l \rightarrow \infty} h^{(l)}=+\infty$. It is easy to see that $U^{(l)}=u^{(l)} / h^{(l)}$ is a solution of the following boundary value problem

$$
\begin{gathered}
\sum_{i, j=1}^{N} a_{i j}^{(l)} U_{x_{i} x_{j}}^{(l)}+\sum_{i=1}^{N} b_{i}^{(l)} U_{x_{i}}^{(l)}+c^{(l)} U^{(l)}=\frac{A^{(l)}}{h^{(l)}} \text { in } Q, \\
\frac{\partial U^{(l)}}{\partial v}+\sigma^{(l)} U^{(l)}=\frac{g^{(l)}}{h^{(l)}} \text { on } \partial Q .
\end{gathered}
$$

Noting that $\sum_{i=1}^{N} b_{i}^{(l)} U_{x_{i}}^{(l)}+c^{(l)} U^{(l)}$ in (2.7) are bounded, and by using the method in Theorem 4.2, Chapter III, [4], we can obtain the estimate

$$
C_{\beta}^{1}\left(U^{(l)}, \bar{Q}\right) \leq M_{4},\left\|U^{(l)}\right\|_{W_{2}^{2}(Q)} \leq M_{5},
$$

where

$\beta(0<\beta \leq \alpha), M_{j}=M_{j}\left(q, p, \beta, k, Q, M_{3}\right)(j=4,5) \quad$ are non-negative constants. Hence from $\left\{U^{(l)}\right\}$, we can choose a subsequence $\left\{U^{\left(l_{k}\right)}\right\}$ such that $\left\{U^{\left(l_{k}\right)}\right\}$, $\left\{U_{x_{i}}^{\left(l_{k}\right)}\right\}$ uniformly converge to $U^{(0)}, U_{x_{i}}^{(0)}$ in $\bar{Q}$ respectively, and $\left\{U_{x_{i} x_{j}}^{\left(l_{k}\right)}\right\}$ weakly converge to $U_{x_{i} x_{j}}^{(0)}$ in $Q$, and $U^{(0)}$ is a solution of the following boundary value problem

$$
\begin{gathered}
\sum_{i, j=1}^{N} a_{i j}^{(0)} U_{x_{i} x_{j}}^{(0)}+\sum_{i=1}^{N} b_{i}^{(0)} U_{x_{i}}^{(0)}+c^{(0)} U^{(0)}=0 \text { on } Q, \\
\frac{\partial U^{(0)}}{\partial v}+\sigma^{(0)} U^{(0)}=0 \text { on } \partial Q .
\end{gathered}
$$

According to Theorem 1.1, we can get $U^{(0)}(x)=0, x \in \bar{Q}$. However, from $C^{1}\left[U^{(l)}, \bar{Q}\right]=1$,
there exists a point $x^{*} \in \bar{Q}$, such that 
$\left|U^{(0)}\left(x^{*}\right)\right|+\sum_{i=1}^{N}\left|U_{x_{i}}^{(0)}\left(x^{*}\right)\right|>0$. This contradiction proves that (2.4) is true. Following the same procedure from (2.4) to (2.9), we can derive the estimates (2.1) and (2.2).

In general we can prove the following theorem. Theorem 2.2. Suppose that Equation (1.2) satisfies Condition $\mathrm{C}^{\prime}$. Then any solution $u(x)$ of Problem $\mathrm{O}$ satisfies the estimates

$$
C_{\beta}^{1}[u, \bar{Q}] \leq M_{6} k_{*},\|u\|_{W_{2}^{2}(Q)} \leq M_{7} k_{*},
$$

where $\beta(0<\beta \leq \alpha), M_{j}=M_{j}\left(q, p, \beta, k_{0}, Q\right)(j=6,7)$ are non-negative constants, $k_{*}=k_{1}+k_{2}+k_{3}$ with $k_{1}$ in (1.5), $k_{2}$ in (1.9), and $k_{3}=k_{0}\left[\sum_{i=1}^{N}\left|u_{x_{i}}\right|^{\sigma_{i}}+|u|^{\sigma_{0}}\right]$.

Proof. If $k_{*}=0$, i.e. $k_{1}=k_{2}=k_{3}=0$, from Theorem 2.1 , it follows that $u(z)=0, z \in Q$. If $k_{*}>0$, it is easy to see that $U(z)=u(z) / k_{*}$ satisfies the complex equation and boundary conditions

$$
\begin{gathered}
\sum_{i, j=1}^{N} a_{i j} U_{x_{i} x_{j}}+\sum_{i=1}^{N} b_{i} U_{x_{i}}+c U=\frac{A-G\left(x, u, D_{x} u\right)}{k_{*}} \text { in } Q, \\
I U=\frac{\partial U}{\partial v}+\sigma U=\frac{g(x)}{k_{*}}, x \in \partial Q .
\end{gathered}
$$

Note that

$L_{p}\left[A_{f} k_{*}, \bar{Q}\right] \leq 1, C_{\alpha}^{1}\left[g(z) / k_{*}, \partial Q\right] \leq 1,|G| / k_{*} \leq 1$. Using a proof similar to that of Theorem 2.1, we have

$$
C_{\beta}^{1}[u, \bar{Q}] \leq M_{8},\|u\|_{W_{2}^{2}(Q)} \leq M_{9} .
$$

From the above estimates, it immediately follows that (2.12) holds.

\section{Solvability of Oblique Derivative Problems for Nonlinear Elliptic Equations of Second Order}

Set $Q_{l}=\{x \in Q \mid \operatorname{dist}(x, \partial Q) \geq 1 / l\}$, where $l$ is a positive integer. We first consider another form of (1.2), namely

$$
\begin{aligned}
& \Delta u=f^{l}\left(x, u, D u, D^{2} u\right), f^{l} \\
& =\Delta u+\sum_{i, j=1}^{N} a_{i j}^{l} u_{x_{i} x_{j}}+\sum_{i=1}^{N} b_{i}^{l} u_{x_{i}}+c^{l} u_{j}-A^{l} \text { in } Q,
\end{aligned}
$$

where the coefficients

$$
a_{i j}^{l}=\left\{\begin{array}{l}
a_{i j}, \\
\delta_{i j},
\end{array} b_{i}^{l}=\left\{\begin{array}{l}
b_{i}, \\
0,
\end{array} c^{l}=\left\{\begin{array}{l}
c, \\
0,
\end{array} A^{l}=\left\{\begin{array}{l}
A \text { in } Q_{l}, \\
0 \text { in } \mathbb{R}^{N} \backslash Q_{l},
\end{array}\right.\right.\right.\right.
$$

Theorem 3.1. Under the same conditions as in Theorem 2.1, if $u(x)$ is a solution of Problem O for (3.1), then $u(x)$ can be expressed in the form

$$
\begin{aligned}
& u(x)=U(x)+V(x)=U(x)+v_{0}(x)+v(x), \\
& v(x)=H \rho=\int_{Q_{0}} G(x-\zeta) \rho(\zeta) \mathrm{d} \zeta, \\
& G= \begin{cases}|x-\zeta|^{2-N} / N(2-N) \omega_{N}, & N>2, \\
\log |x-\zeta| / 2 \pi, & N=2,\end{cases}
\end{aligned}
$$

where $\rho(x)=\Delta u, Q_{0}=\{|x|<R\}$ with a large here $R$ such that $Q_{0} \supset \bar{Q}$, and $\omega_{N}=2 \pi^{N / 2} / N \Gamma(N / 2)$ is the volume of the unit ball in $\mathbb{R}^{N}$. In the expression, $V(x)$ is a solution of Problem $\mathrm{D}_{0}$ for (3.1), namely the Equation (3.1) in $Q_{0}$ with the boundary condition $V(x)=0$ on $\partial Q_{0}$. And $U(x)$ is a solution of Problem $\mathrm{O}$ for $\Delta U=0$ in $Q$ with the boundary condition (3.11) below, which satisfies the estimates

$$
\hat{C}_{\beta}^{1}[U, \bar{Q}]+\|U\|_{W_{2}^{2}(Q)} \leq M_{10}, C_{\beta}^{1}\left[V, \overline{Q_{0}}\right]+\|V\|_{W_{2}^{2}\left(Q_{0}\right)} \leq M_{11} \text {, }
$$

where $\beta(0<\beta \leq \alpha), M_{j}=M_{j}\left(q, p, \beta, k, Q_{l}\right)(j=10,11)$ are non-negative constants with $q=\left(q_{0}, q_{1}\right)$ and $k=\left(k_{0}, k_{1}, k_{2}\right)$.

Proof. It is easy to see that the solution $u(x)$ of Problem $O$ for Equation (3.1) can be expressed by the form (3.3). Noting that $a_{i j}^{l}=0(i \neq j), \quad b_{i}^{l}=0, \quad c^{l}=0$, $A^{l}(x)=0$ in $\mathbb{R}^{N} \backslash Q_{l}$ and $V(x)$ is a solution of Problem $\mathrm{D}_{0}$ for (3.1) in $Q_{0}$, we can obtain that $V(x)$ in $\hat{Q}_{2 l}=\bar{Q} \backslash Q_{2 l}$ satisfies the estimate

$$
C^{2}\left[V(x), \hat{Q}_{21}\right] \leq M_{12}=M_{12}\left(q, p, \beta, k, Q_{l}\right) .
$$

On the basis of Theorem 2.1, we can see that $U(x)$ satisfies the first estimate in (3.4), and then $V(x)$ satisfies the second estimate in (3.4).

Theorem 3.2. If Equation (1.2) satisfies the same conditions as in Theorem 2.1, then Problem O for (3.1) has a solution $u(x)$.

Proof. We prove the existence of solutions of Problem $\mathrm{O}$ for the nonlinear Equation (3.1) by using the LeraySchauder theorem. Introduce the equation with the parameter $h \in[0,1]$ :

$$
\Delta u=h f^{l}\left(x, u, D u, D^{2} u\right) \text { in } Q .
$$

Denote by $B_{M}$ a bounded open set in the Banach space $\hat{W}_{2}^{2}\left(Q_{0}\right)=C_{\beta}^{1}\left(\bar{Q}_{0}\right) \cap W_{2}^{2}\left(Q_{0}\right)(0<\beta \leq \alpha)$, the elements of which are real functions $V(x)$ satisfying the inequalities

$$
\|V\|_{\hat{W}_{2}^{2}\left(Q_{0}\right)}=C_{\beta}^{1}\left[V, \overline{Q_{0}}\right]+\|V\|_{W_{2}^{2}\left(Q_{0}\right)}<M_{13}=M_{11}+1,
$$

with the non-negative constant $M_{11}$ as stated in (3.4). We choose any function $\tilde{V}(x) \in \overline{B_{M}}$ and substitute it into the appropriate positions on the right hand side of (3.5), and form an integral $\tilde{v}(x)=H \rho$ as follows:

$$
\tilde{v}(x)=H \tilde{\rho}, \tilde{\rho} x=\Delta \tilde{V} .
$$

Let $\tilde{v}_{0}(x)$ be a solution of the boundary value prob- 
lem in $Q_{0}$ :

$$
\begin{gathered}
\Delta \tilde{v}_{0}=0 \text { on } Q_{0}, \\
\tilde{v}_{0}(x)=-\tilde{v}(x) \text { on } \partial Q_{0},
\end{gathered}
$$

and denote by $\hat{V}(x)=\tilde{v}(x)+\tilde{v}_{0}(x)$ the solution of the corresponding Problem $\mathrm{D}_{0}$. Moreover on the basis of the result in [4], we can find a solution $\tilde{U}(x)$ of the corresponding Problem $\tilde{O}$ in $Q$ :

$$
\Delta \tilde{U}=0 \text { on } Q,
$$

$$
\frac{\partial \tilde{U}}{\partial v}+\sigma(x) \tilde{U}=g(x)-\frac{\partial \hat{V}}{\partial v}+\sigma(x) \hat{V} \text { on } \partial Q .
$$

Now consider the equation

$$
\Delta V=h f^{l}\left(x, \tilde{u}, D \tilde{u}, D^{2} \tilde{U}+D^{2} V\right), 0 \leq h \leq 1,
$$

where $\tilde{u}=\tilde{U}+\hat{V}$. By Condition $\mathrm{C}$ and the principle of contracting mapping, we can find a unique solution $V(x)$ of Problem $\mathrm{D}_{0}$ for Equation (3.12) in $Q_{0}$ satisfying the boundary condition

$$
V(x)=0 \text { on } \partial Q_{0} .
$$

Denote $u(x)=U(x)+V(x)$, where $U$ is obtained from $V$ in the same way as getting $\tilde{U}$ from $\tilde{V}$. Denote by $V=S(\tilde{V}, h), u=S_{1}(\tilde{V}, h)(0 \leq h \leq 1)$ the mappings from $\tilde{V}$ onto $V$ and $u$ respectively. Furthermore, if $V(x)$ is a solution of Problem $O$ in $Q_{0}$ for the equation

$$
\Delta V=h f^{l}\left(x, u, D u, D^{2}(U+V)\right), 0 \leq h \leq 1,
$$

where $u=S_{1}(V, h)$, then from Theorem 3.1, $V(x)$ satisfies the second estimate in (3.4), consequently $V(x) \in B_{M}$. Set $B_{0}=B_{M} \times[0,1]$. We can verify that the mapping $V=S(\tilde{V}, h)$ satisfies the three conditions of Leray-Schauder theorem:

1) For every $h \in[0,1], V=S(\tilde{V}, h)$ continuously maps the Banach space $B$ into itself, and is completely continuous on $B_{M}$. Besides, for every function $\tilde{V}(x) \in \overline{B_{M}}, S(\tilde{V}, h)$ is uniformly continuous with respect to $h \in[0,1]$.

2) For $h=0$, from (3.6) and (3.12), it is clear that $V=S(\tilde{V}, 0) \in B_{M}$.

3) From Theorems 3.1 , we know that $V=S(\tilde{V}, h)(0 \leq h \leq 1)$ satisfies the second estimate in (3.4). Moreover by the inequality (3.6), it is not difficult to see that the functional equation

$V=S(\tilde{V}, h)(0 \leq h \leq 1)$ does not have any solution $V(x)$ on the boundary $\partial B_{M}=\overline{B_{M}} \backslash B_{M}$.

Hence by the Leray-Schauder theorem, we know that Problem $\mathrm{D}_{0}$ for (3.12) with $h=1$ has a solution $V(x) \in B_{M}$, and then Problem $\mathrm{O}$ of system (3.5) with $h=1$, i.e. (3.1) has a solution

$$
\begin{aligned}
u(x) & =S_{1}(V, h)=U(x)+V(x) \\
& =U(x)+v_{0}(x)+v(x) \in B .
\end{aligned}
$$

Theorem 3.3. Under the same conditions as in Theorem 2.1, Problem O for the Equation (1.2) has a solution.

Proof. By Theorems 2.1 and 3.2, Problem O for (3.1) possesses a solution $u^{l}(x)$, which satisfies the estimates (2.1) and (2.2), where $l=1,2, \cdots$. Thus, we can choose a subsequence $\left\{u^{l_{k}}(x)\right\}$, such that $\left\{u^{l_{k}}(x)\right\},\left\{u_{x_{i}}^{l_{k}}(x)\right\}(i=1, \cdots, N)$ in $\bar{Q}$ uniformly converge to $u^{0}(x), u_{x_{i}}^{0}(x)(i=1, \cdots, N)$ respectively. Obviously, $u^{0}(x)$ satisfies the boundary condition of Problem O. On the basis of the principle of compactness of solutions for (3.1), it is easy to see that $u^{0}(x)$ is a solution of Problem $O$ for (1.2). Next we provide a further discussion on the Equation (1.2), namely

$$
\begin{aligned}
& F\left(x, u, D_{x} u, D_{x}^{2} u\right)=-G\left(x, u, D_{x} u\right) \text { in } Q, \\
& F\left(x, u, D_{x} u, D_{x}^{2} u\right)=\sum_{i, j=1}^{N} a_{i j} u_{x_{i} x_{j}}+\sum_{i=1}^{N} b_{i} u_{x_{i}}+c u-A
\end{aligned}
$$

with the boundary condition

$$
\frac{\partial u}{\partial v}+\sigma(x) u=g(x) \text { on } \partial Q .
$$

Theorem 3.4. Let the complex Equation (3.15) satisfy Condition $\mathrm{C}^{\prime}$.

1) When $0<\sigma_{0}, \sigma_{1}, \cdots, \sigma_{N}<1$, Problem $O$ for (3.15) has a solution $u(x) \in W_{p_{0}}^{2}(Q)$, where $p_{0}$ is a constant as stated before.

2) When $\min \left(\sigma_{0}, \sigma_{1}, \cdots, \sigma_{N}\right)>1$, Problem $\mathrm{O}$ for (3.15) has a solution $u(x) \in W_{p_{0}}^{2}(Q)$, provided that

$$
M_{14}=L_{p}[A, \bar{Q}]+C_{\alpha}^{2}[g, \partial Q]
$$

is sufficiently small.

Proof. 1) Consider

$$
\begin{aligned}
& \left(M_{6}+M_{7}\right)\left\{L_{p}[A, \bar{Q}]+\sum_{i=1}^{N} L_{\infty}\left[B_{i}, \bar{Q}\right] t^{\sigma_{i}}\right. \\
& \left.+L_{\infty}\left[B_{0}, \bar{Q}\right] t^{\sigma_{0}}+C_{\alpha}^{2}[g, \partial Q]\right\}=t,
\end{aligned}
$$

where $M_{6}, M_{7}$ are positive constants as in (2.12). Because $0<\sigma_{0}, \sigma_{1}, \cdots, \sigma_{N}<1$, the above equation has a unique solution $t=M_{15}>0$. Now we introduce a bounded, closed and convex subset $B^{*}$ of the Banach space $C^{1}(\bar{Q}) \cap W_{2}^{2}(\bar{Q})$ as follows:

$$
B^{*}=\left\{u(x) \in C^{1}(\bar{Q}) \cap W_{2}^{2}(\bar{Q}) \mid C^{1}(u, \bar{Q}]+\|u\|_{W_{2}^{2}(Q)} \leq M_{15}\right\} .
$$

We choose any functions $\tilde{u}(x) \in B^{*}$ and substitute it into the appropriate positions in two functions $F\left(x, u, D_{x} u, D_{x}^{2} u\right), G\left(x, u, D_{x} u\right)$ in (3.15) and the 
boundary condition (3.16), and obtain the following

$$
\begin{gathered}
\tilde{F}\left(x, \tilde{u}, D_{x} \tilde{u}, D_{x}^{2} \tilde{u}, u, D_{x} u, D^{2} u\right) \\
+G\left(x, \tilde{u}, D_{x} \tilde{u}, u, D_{x} u\right)=0 \text { in } Q, \\
\quad \frac{\partial u}{\partial v}+\sigma(x) u=g(x) \text { on } \partial Q .
\end{gathered}
$$

where

$$
\begin{aligned}
& \tilde{F}\left(x, \tilde{u}, D_{x} \tilde{u}, D_{x}^{2} \tilde{u}, u, D_{x} u, D^{2} u\right)=\sum_{i, j=1}^{N} a_{i j}\left(x, \tilde{u}, \tilde{u}_{x}, \tilde{u}_{x_{i} x_{j}}\right) u_{x_{i} x_{j}} \\
& +\sum_{i=1}^{N} b_{i}\left(x, \tilde{u}, \tilde{u}_{x}\right) u_{x_{i}}+c(x, \tilde{u}) u-A, \\
& G\left(x, \tilde{u}, D_{x} \tilde{u}, u, D_{x} u\right)=\sum_{i=1}^{N} B_{i}\left(\tilde{u}, D_{x} \tilde{u}\right)\left|u_{x_{i}}\right|^{\sigma_{i}} \\
& +B_{0}\left(\tilde{u}, D_{x} \tilde{u}\right)|u|^{\sigma_{0}} .
\end{aligned}
$$

In accordance with the method in the proof of Theorem 3.2, we can prove that the boundary value problem (3.20), (3.21) has a unique solution $u(x)$. Denote by $u(x)=T[\tilde{u}(x)]$ the mapping from $[\tilde{u}(x)]$ to $[u(x)]$. Noting that

$$
L_{p}\left[\sum_{i=1}^{N} b_{i} \tilde{u}_{x_{i}}, \partial Q\right], C_{\alpha}[c \tilde{u}, \Gamma] \leq M_{6} k_{0}\left(k_{1}+k_{2}+k_{3}\right),
$$

from Theorem 2.2, we have

$$
\begin{aligned}
C^{1}[u, \bar{Q}]+\|u\|_{W_{2}^{2}(Q)} \\
\leq\left(M_{6}+M_{7}\right)\left\{L_{p}[A, \bar{Q}]+C_{\alpha}^{2}[g, D]+L_{\infty}[G, \bar{Q}]\right\} \\
\leq\left(M_{6}+M_{7}\right)\left\{M_{14}+\sum_{i=1}^{N} L_{\infty}\left[B_{i}, \bar{Q}\right] C\left[\tilde{u}_{x_{i}} \bar{Q}\right]^{\sigma_{i}}\right. \\
\left.\quad+L_{\infty}\left[B_{0}, \bar{Q}\right] C[\tilde{u}, \bar{Q}]^{\sigma_{0}}\right\} \\
\leq\left(M_{6}+M_{7}\right)\left\{M_{14}+k_{0} \sum_{i=1}^{N} M_{15}^{\sigma_{i}}+k_{0} M_{15}^{\sigma_{0}}\right\}=M_{15} .
\end{aligned}
$$

This shows that $T$ maps $B^{*}$ onto a compact subset in $B^{*}$. Next, we verify that $T$ in $B^{*}$ is a continuous operator. In fact, we arbitrarily select a sequence $\left\{\tilde{u}_{n}(z)\right\}$ in $B^{*}$, such that

$$
C^{1}\left(\tilde{u}_{n}-\tilde{u}_{0}, \bar{Q}\right)+\left\|\tilde{u}_{n}-\tilde{u}_{0}\right\|_{W_{2}^{2}(Q)} \rightarrow 0 \text { as } n \rightarrow \infty .
$$

By Theorem 2.1, we can derive that

$$
\begin{aligned}
& L_{p}\left[\tilde{F}\left(x, \tilde{u}_{n}, D_{x} \tilde{u}_{n}, x, u_{0}, D_{x} u_{0}, D_{x}^{2} u_{0}\right)\right. \\
& \left.-\tilde{F}\left(x, \tilde{u}_{0}, D_{x} \tilde{u}_{0}, D^{2} \tilde{u}_{0}, u_{0}, D_{x} u_{0}, D^{2} u_{0}\right), \bar{Q}\right] \rightarrow 0 \text { as } n \rightarrow \infty,
\end{aligned}
$$

in which $u_{0}(x) \in C^{1}(\bar{D}) \cap W_{2}^{2}(Q)$.

Moreover, from $u_{n}=T\left[\tilde{u}_{n}\right], u_{0}=T\left[\tilde{u}_{0}\right]$, it is clear that $u_{n}-u_{0}$ is a solution of Problem O for the following equation

$$
\begin{aligned}
& \tilde{F}\left(x, \tilde{u}_{n}, D_{x} \tilde{u}_{n}, D_{x}^{2} \tilde{u}_{n}, u_{0}, D_{x} u_{0}, D_{x}^{2} u_{0}\right) \\
& -\tilde{F}\left(x, \tilde{u}_{0}, D_{x} \tilde{u}_{0}, D_{x}^{2} \tilde{u}_{0}, u_{0}, D_{x} u_{0}, D_{x}^{2} u_{0}\right) \\
& +G\left(x, \tilde{u}_{n}, D_{x} \tilde{u}_{n}, u_{0}, D_{x} u_{0}\right) \\
& -G\left(x, \tilde{u}_{0}, D_{x} \tilde{u}_{0}, u_{0}, D_{x} u_{0}\right)=0 \text { in } Q,
\end{aligned}
$$

$$
\frac{\partial\left(u_{n}-u_{0}\right)}{\partial v}+\sigma(x)\left(u_{n}-u_{0}\right)=0 \text { on } \partial Q \text {. }
$$

In accordance with the method in the proof of Theorem 2.2, we can obtain the estimate

$$
\begin{aligned}
C^{1} & {\left[u_{n}-u_{0}, \bar{Q}\right]+\left\|u_{n}-u_{0}\right\|_{W_{2}^{2}(Q)} } \\
\leq & M_{16}\left\{L _ { p } \left[G\left(x, \tilde{u}_{n}, D_{x} \tilde{u}_{n}, u_{0}, D_{x} u_{0}\right)\right.\right. \\
& \left.-G\left(x, \tilde{u}_{0}, D_{x} \tilde{u}_{0}, u_{0}, D_{x} u_{0}\right), \bar{Q}\right] \\
& +L_{p}\left[\tilde{F}\left(x, \tilde{u}_{n}, D_{x} \tilde{u}_{n}, D_{x}^{2} \tilde{u}_{n}, u_{0}, D_{x} u_{0}, D_{x}^{2} u_{0}\right)\right. \\
& \left.\left.-\tilde{F}\left(x, \tilde{u}_{0}, D_{x} \tilde{u}_{0}, D_{x}^{2} \tilde{u}_{0}, u_{0}, D_{x} u_{0}, D_{x}^{2} u_{0}\right), \bar{Q}\right]\right\},
\end{aligned}
$$

in which $M_{16}=M_{16}\left(q_{0}, p_{0}, \beta, k_{0}, Q\right)$. From (2.12) and the above estimate, we obtain

$C^{1}\left[u_{n}-u_{0}, \bar{Q}\right]+\left\|u_{n}-u_{0}\right\|_{W_{2}^{2}(Q)} \rightarrow 0$ as $n \rightarrow \infty$. On the basis of the Schauder fixed-point theorem, there exists a function $u(x) \in C^{1}(\bar{Q}) \cap W_{2}^{2}(Q)$ such that $u(x)=T[u(x)]$. It is clear that $u(x)$ is a solution of Problem $\mathrm{O}$ for the Equation (3.15) and the boundary condition (3.16) with $0<\sigma_{0}, \cdots, \sigma_{N}<1$.

2) Secondly, we discuss the case:

$\min \left(\sigma_{0}, \cdots, \sigma_{N}\right)>1$. In this case, (3.18) has the solution $t=M_{16}$ provided that $M_{14}$ in (3.17) is small enough. Consider a closed and convex subset $B_{*}$ in the Banach space $C^{1}(\bar{Q}) \cap W_{2}^{2}(Q)$, i.e.

$$
B_{*}=\left\{u(x) \in C^{1}(\bar{Q}) \cap W_{2}^{2}(Q) \mid C^{1}[u, \bar{Q}]+\|u\|_{W_{2}^{2}(Q)} \leq M_{16}\right\} .
$$

Applying a method similar to the one in (1), we can verify that there exists a solution $u(x) \in C^{1}(\bar{Q}) \cap W_{2}^{2}(Q)$ of Problem O for (3.15) when $\min \left(\sigma_{0}, \sigma_{1}, \cdots, \sigma_{N}\right)>1$.

Note: The opinions expressed herein are those of the authors and do not necessarily represent those of the Uniformed Services University of the Health Sciences and the Department of Defense.

\section{REFERENCES}

[1] H. O. Codres, "Über die erste Randwertaufgabe bei quasilinearen Differentialgleichungen zweiter Ordnung in mehr als zwei Variablen," Mathematische Annalen, Vol. 131, No. 3, 1956, pp. 278-312. http://dx.doi.org/10.1007/BF01342965 
[2] Y. A. Alkhutov and I. T. Mamedov, "The First Boundary Value Problem for Nondivergence Second Order Parabolic Equations with Discontinuous Coefficients," Mathematics of the USSR-Sbornik, Vol. 59, No. 2, 1988, pp. 471-495.

http://dx.doi.org/10.1070/SM1988v059n02ABEH003147

[3] O. A. Ladyshenskaja and N. N. Uraltseva, "Linear and Quasilinear Elliptic Equations," Academic Press, New York, 1968.

[4] G. C. Wen, Z. L. Xu and H. Y. Gao, "Boundary Value Problems for Nonlinear Elliptic Equations in High Dimensional Domains," Research Information Ltd., Slough, 2004.

[5] G. C. Wen, D. Chen and Z. L. Xu, "Nonlinear Complex Analysis and Its Applications," Science Press, Beijing, 2008.
[6] G. C. Wen, "Recent Progress in Theory and Applications of Modern Complex Analysis," Science Press, Beijing, 2010 .

[7] G. C. Wen and D. Chen, "Oblique Derivative Problems for Nonlinear Elliptic Equations of Second Order with Measurable Coefficients in High Dimensional Domains," Nonlinear Sciences and Numerical Simulation, Vol. 10 No. 5, 2005, pp. 559-570. http://dx.doi.org/10.1016/i.cnsns.2003.04.002

[8] D. Chen and G. C. Wen, "Some Boundary Value Problems for Nonlinear Elliptic Systems of Second Order in High Dimensional Domains," Proceedings of the 3rd International Conference on Boundary Value Problems, Integral Equations and Related Problems, World Scientific, Singapore, 2011, pp. 12-21. 